\title{
Medium Voltage Resonant Converter with Balanced Input Capacitor Voltages and Output Diode Currents
}

\author{
Bor-Ren $\operatorname{Lin}^{\dagger}$ and Yan-Kang Du* \\ ${ }^{+*}$ Department of Electrical Engineering, National Yunlin University of Science and Technology, Yunlin, Taiwan
}

\begin{abstract}
This paper presents a $1.92 \mathrm{~kW}$ resonant converter for medium voltage applications that uses low voltage stress MOSFETs $(500 \mathrm{~V})$ to achieve zero voltage switching (ZVS) turn-on. In the proposed converter, four MOSFETs are connected in series to limit the voltage stress of the power switches at half of the input voltage. In addition, three resonant circuits are adopted to share the load current and to reduce the current stress of the passive components. Furthermore, the transformer primary and secondary windings are connected in series to balance the output diode currents for medium power applications. Split capacitors are adopted in each resonant circuit to reduce the current stress of the resonant capacitors. Two balance capacitors are also used to automatically balance the input capacitor voltage in every switching cycle. Based on the circuit characteristics of the resonant converter, the MOSFETs are turned on under ZVS. If the switching frequency is less than the series resonant frequency, the rectifier diodes can be turned off under zero current switching (ZCS). Experimental results from a prototype with a 750-800 V input and a $48 \mathrm{~V} / 40 \mathrm{~A}$ output are provided to verify the theoretical analysis and the effectiveness of the proposed converter.
\end{abstract}

Key words: Interleaved PWM, PWM converters, Switching mode power supplies

\section{INTRODUCTION}

Full-bridge converters with a simple structure, constant frequency pulse-width modulation and soft switching turn-on [1]-[4] have been adopted in medium power applications. However, power switches should suffer the input voltage due to the full-bridge structure. High input DC/DC converters have been developed for ship electric power distribution systems [5], three-phase AC/DC converters and the traction systems for light trains [6]-[7]. Three-level DC/DC converters [8]-[10] with low voltage and current stresses were presented to reduce the voltage stress on active switches. However, the power switches are operated at hard switching. High switching losses on the power switches reduce the circuit efficiency. In order to achieve soft switching and reduce switching losses, three-level zero voltage switching (ZVS) converters for high input voltage applications were proposed in [11]-[14]. In [13], four power switches with $V_{\text {in }} / 2$ voltage stress and two power switches with $V_{\text {in }}$ voltage stress

Manuscript received Aug. 27, 2014; accepted Nov. 7, 2014

Recommended for publication by Associate Editor Joung-Hu Park.

${ }^{\dagger}$ Corresponding Author: linbr@yuntech.edu.tw

Tel: +886-5-5516128, Fax: +886-05-342601 ext. 4259, YunTech

*Dept. of Electrical Eng., National Yunlin University of Science and Technology, Taiwan are used in the three-level ZVS hybrid full bridge converter. The drawback of this circuit is that two power switches with high voltage stress are used in this converter. Resonant converters [15]-[18] have been proposed for the advantages of high conversion efficiency and high power density. Power switches are turned on under ZVS from low load to full load. If the switching frequency is less than the series resonant frequency, the rectifier diodes at output side are turned off under zero current switching (ZCS). The series-parallel connection techniques have been discussed in [19]-[21]. In [19], two transformers are adopted in three-level converter with current double rectifier for medium voltage application. However, the high current rating of rectifier diodes and large size of filter inductors are needed in this circuit topology for high load current application. The circuit efficiency will be drop at light load due to the high circulating current in conventional three-level PWM converter. The input split capacitor voltages cannot be automatically balanced. An active clamp forward converter with parallel connection in primary side was presented in [20] to achieve soft switching. However, this circuit topology cannot be used for medium voltage applications due to its high voltage stress on the power switches. A three-level resonant converter with duty cycle control has been presented in [21] to have ZVS turn-on 
for all of the power switches and ZCS turn-off for all of the rectifier diodes. The output currents of the two secondary sides are automatically balanced due to the series connection of the transformers. However, the input split capacitor voltages cannot be balanced automatically and the duty cycle is decreased under a light load case. Thus, the circuit efficiency under a light load is decreased.

A ZVS DC/DC converter for high input voltage and load current applications is studied in this paper. Two half-bridge legs with split capacitors are connected in series at the high voltage side to clamp the voltage stress of the power switches at half of the input voltage. Two balance capacitors are used between the AC sides of the two half-bridge legs to automatically balance the input split capacitor voltages. Three resonant circuits are used at the high voltage side in order to reduce the current stress of the resonant components. The secondary windings of the transformers are connected in series to automatically balance the output diode currents. Since the input impedance of the resonant tank is an inductive load at the switching frequency, the power switches are turned on under ZVS. As a result, the switching losses on the power switches are reduced. Finally, experiments from a 750-800 V input and 48V/40A output prototype were provided to verify the performance of the proposed converter.

\section{PROPOSED CONVERTER AND OPERATION PRINCIPLE}

For three-phase power factor corrector converters with a $380 \mathrm{~V}$ or $480 \mathrm{~V}$ utility voltage or a DC traction system, the input voltage of the DC/DC converter is equal to or higher than $750 \mathrm{~V}$. Fig. 1 gives the circuit topology of the proposed converter for medium voltage applications. The circuit components at the high voltage side include the input voltage $V_{\text {in }}$, power MOSFETs $S_{1}-S_{4}$ with their body diodes and parallel capacitors $C_{o s s 1}-C_{o s s 4}$, resonant capacitors $C_{r 1}-C_{r 6}$, resonant inductors $L_{r 1}-L_{r 3}$, and transformers $T_{1}-T_{4}$. Two center-tapped rectifiers are used at the low voltage side to share the load current and to reduce the current stress of the passive components. The secondary windings of $T_{1}$ and $T_{3}$ are connected in series so that the primary currents $i_{L r 1}$ and $i_{L r 3}$ are balanced. Similarly, the primary currents $i_{L r 2}$ and $i_{L r 3}$ are balanced since the secondary windings of $T_{2}$ and $T_{4}$ are connected in series. Thus, the primary and secondary winding currents of $T_{1}-T_{4}$ are balanced. $\left(S_{1}\right.$ and $\left.S_{3}\right)$ and $\left(S_{2}\right.$ and $\left.S_{4}\right)$ have the same PWM waveforms with a 0.5 duty cycle. However, the driving signals of $S_{2}$ and $S_{4}$ are complementary with the driving signals of $S_{1}$ and $S_{3}$ with a short dead time. The components $S_{1}-S_{4}$ and $C_{r 1}-C_{r 6}$ establish a switched capacitor circuit [22]. Therefore, the input capacitor voltages are balanced, $v_{C r 1}+v_{C r 2}=v_{C r 3}+v_{C r 4}=v_{C r 5}+v_{C r 6}=V_{\text {in }} / 2$. The proposed converter includes three resonant circuits at the high voltage side. A variable frequency scheme is adopted to

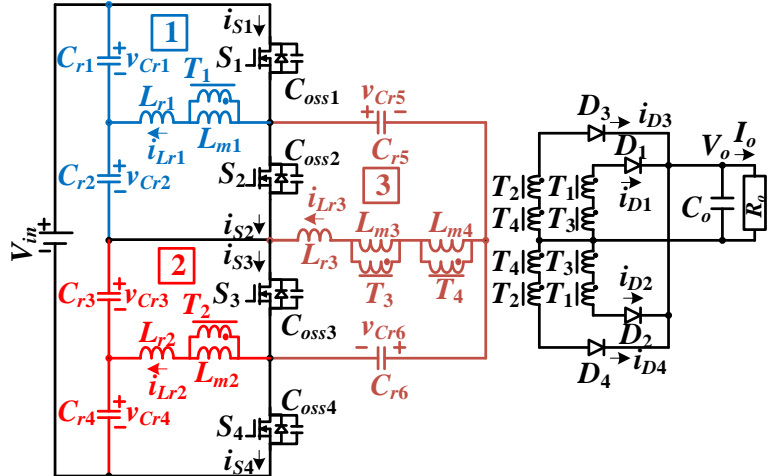

Fig. 1. Circuit configuration of the proposed converter.

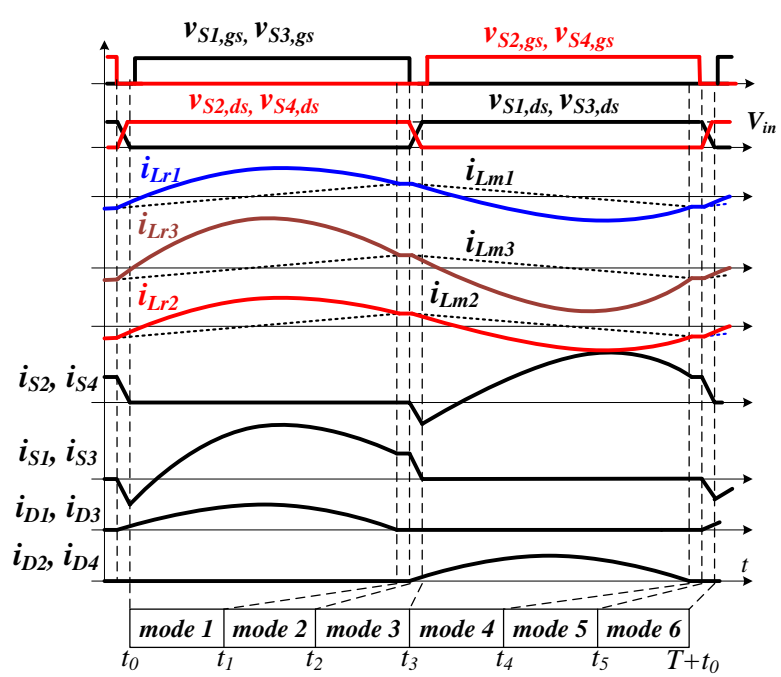

Fig. 2. Key waveforms of the proposed converter.

regulate the output voltage.

The following assumptions are presumed to simplify the system analysis of the three resonant circuits. The transformers $T_{1}-T_{4}$ have the magnetizing inductances $L_{m 1}=L_{m 2}=2 L_{m 3}=2 L_{m 4}=L_{m}$ and the turns ratios $n_{1}=n_{2}=2 n_{3}=2 n_{4}=n$. The resonant capacitances are $C_{r 1}=C_{r 2}=$ $C_{r 3}=C_{r 4}=C_{r 5}=C_{r 6}=C_{r}$. The resonant inductances are identical $L_{r 1}=L_{r 2}=L_{r 3}=L_{r}$. The power MOSFETs $S_{1}-S_{4}$ have the same output capacitances $C_{o s s 1}=C_{o s s 2}=C_{o s s 3}=C_{o s s 4}=C_{o s s}$. The capacitor voltages $v_{C r 1}+v_{C r 2}=v_{C r 3}+v_{C r 4}=v_{C r 5}+v_{C r 6}=V_{i n} / 2$. If the switching frequency $f_{s w}$ is less than the series resonant frequency $f_{r}$, there are six operating modes in a switching cycle. The main PWM waveforms of the proposed converter under $f_{s w}<f_{r}$ are shown in Fig. 2 and Fig. 3. They give the corresponding equivalent circuit for each operation mode. If the switching frequency $f_{s w}>f_{r}$, then the resonant circuit has only four operation modes (modes 1, 3, 4 and 6) in a switching cycle. In the following statements, the six modes of operation are discussed in each switching cycle. Before time $t_{0}, S_{1}-S_{4}, D_{2}$ and $D_{4}$ are in the off-state. $C_{o s s 1}$ and $C_{\text {oss3 }}$ are discharged, and $C_{\text {oss } 2}$ and $C_{\text {oss } 4}$ are charged.

Mode 1 [ $\boldsymbol{t}_{\boldsymbol{0}}-\boldsymbol{t}_{\mathbf{1}}$ ]: Mode 1 starts at time $t_{0}$ when $C_{\text {oss } 1}$ and $C_{\text {oss } 3}$ are discharged to zero voltage. Since $i_{L r 1}\left(t_{0}\right)<0, i_{L r 2}\left(t_{0}\right)<0$ and 


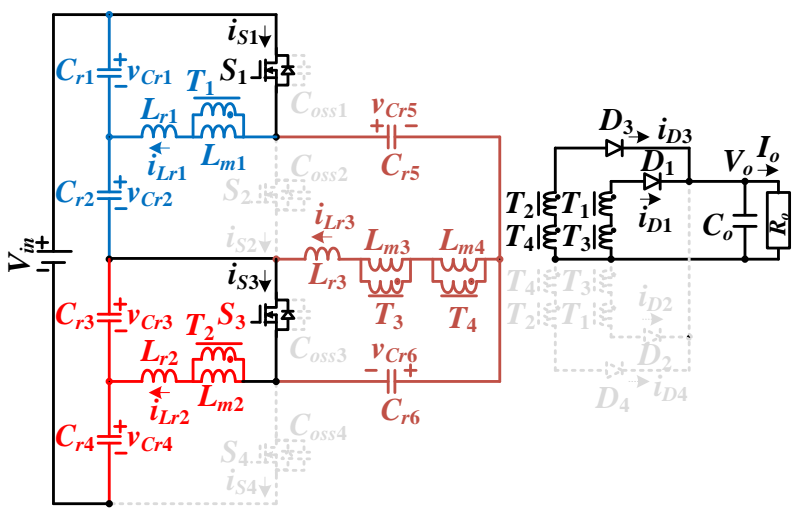

(a)

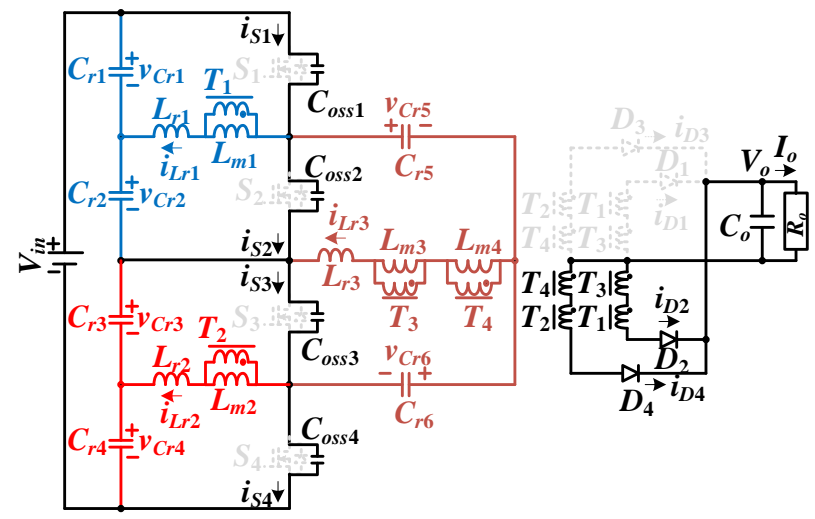

(c)

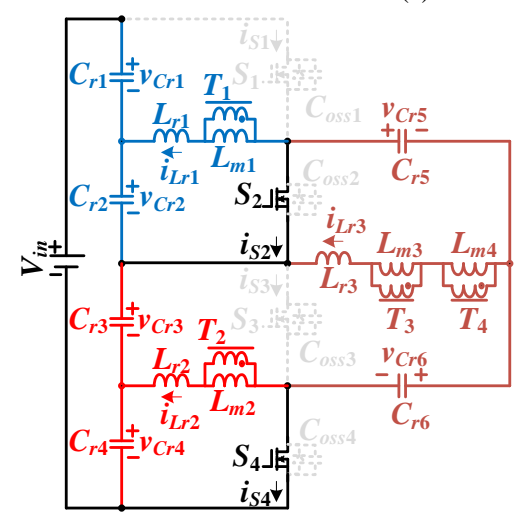

(e)

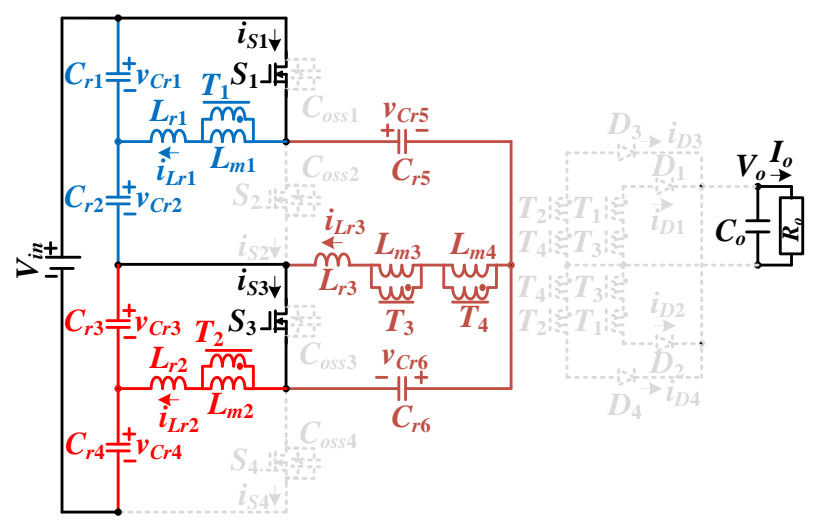

(b)

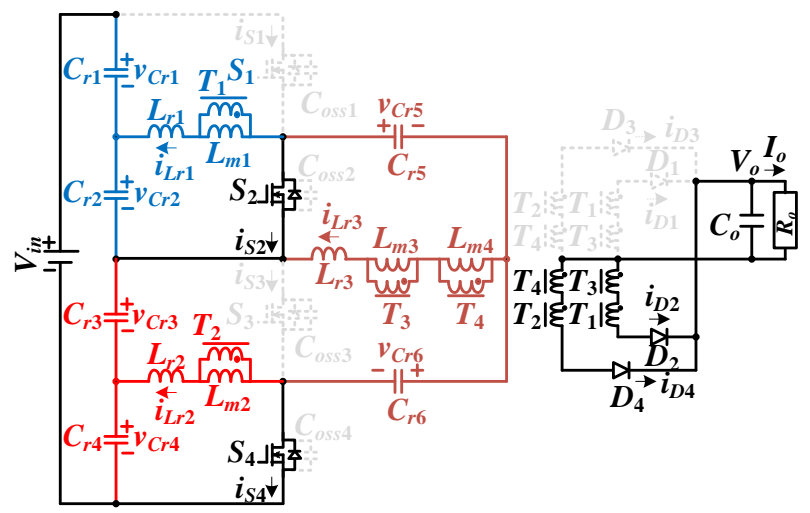

(d)

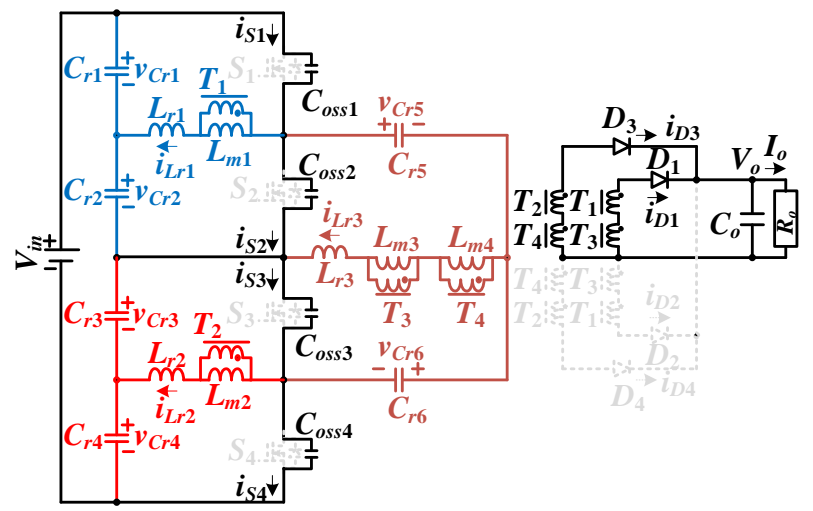

(f)

Fig. 3. Operation modes of the proposed converter in a switching cycle. (a) Mode 1. (b) Mode 2. (c) Mode 3. (d) Mode 4. (e) Mode 5. (f) Mode 6.

$i_{L r 3}\left(t_{0}\right)<0$, the anti-parallel diodes of $S_{1}$ and $S_{3}$ are conducting. Thus, $S_{1}$ and $S_{3}$ are turned on at this moment under ZVS. The voltage stresses of $S_{2}$ and $S_{4}$ are equal to $v_{C r 1}+v_{C r 2}$ and $v_{C r 3}+v_{C r 4}$, respectively. In this mode, the capacitor voltage $v_{C r 5}+v_{C r 6}=v_{C r 1}+v_{C r 2}$. On the other hand, the capacitor voltage $v_{C r 5}+v_{C r 6}=v_{C r 3}+v_{C r 4}$ in mode 3 . Thus, it can be obtained that $v_{C r 1}+v_{C r 2}=v_{C r 3}+v_{C r 4}=v_{C r 5}+v_{C r 6}=V_{\text {in }} / 2$ in steady state. Since $i_{L r 1}>i_{L m 1}, i_{L r 2}>i_{L m 2}$ and $i_{L r 3}>i_{L m 3}$, diodes $D_{1}$ and $D_{3}$ are conducting. In this mode, $v_{L m 1}-v_{L m 4}$ are positive and $i_{L m 1}-i_{L m 5}$ increase. In addition, $L_{r 1}, C_{r 1}$ and $C_{r 2}$ are resonant in circuit $1 ; L_{r 2}, C_{r 3}$ and $C_{r 4}$ are resonant in circuit 2; $L_{r 3}, C_{r 5}$ and $C_{r 6}$ are resonant in circuit 3; and power is transferred from the input voltage $V_{\text {in }}$ to the output load $R_{o}$. The resonant frequency is $f_{r}=1 / 2 \pi \sqrt{2 L_{r} C_{r}}$.

Mode $2\left[\boldsymbol{t}_{\boldsymbol{1}}-\boldsymbol{t}_{2}\right]$ : At time $t_{1}, i_{L r 1}\left(t_{1}\right)=i_{L m 1}\left(t_{1}\right), i_{L r 2}\left(t_{1}\right)=i_{L m 2}\left(t_{1}\right)$ and $i_{L r 3}\left(t_{1}\right)=i_{L m 3}\left(t_{1}\right)=i_{L m 4}\left(t_{1}\right)$. Thus, diodes $D_{1}-D_{4}$ are all in the off-state. In this mode, $L_{r 1}, L_{m 1}, C_{r 1}$ and $C_{r 2}$ are resonant in circuit $1 ; L_{r 2}, L_{m 2}, C_{r 3}$ and $C_{r 4}$ are resonant in circuit 2; and $L_{r 3}, L_{m 3}, L_{m 4}, C_{r 5}$ and $C_{r 6}$ are resonant in circuit 3 . The resonant frequency is $f_{p}=1 / 2 \pi \sqrt{2\left(L_{r}+L_{m}\right) C_{r}}$.

Mode 3 [ $\left.\boldsymbol{t}_{2}-\boldsymbol{t}_{3}\right]$ : At time $t_{2}, S_{1}$ and $S_{3}$ are turned off. Diodes $D_{2}$ and $D_{4}$ are conducting, $v_{L m 1}-v_{L m 4}$ are negative, and $i_{L m 1}-$ $i_{L m 4}$ decrease. Since $i_{L r 1}\left(t_{2}\right)>0, i_{L r 2}\left(t_{2}\right)>0$ and $i_{L r 3}\left(t_{2}\right)>0, C_{o s s 1}$ 
and $C_{\text {oss } 3}$ are charged and $C_{\text {oss } 2}$ and $C_{\text {oss } 4}$ are discharged. $C_{\text {oss2 }}$ and $C_{\text {oss } 4}$ can be discharged to zero voltage if the energy stored in $L_{r 1}-L_{r 3}$ at $t_{2}$ is greater than the energy stored in $C_{\text {oss } 1}-C_{\text {oss } 4}$.

Mode $4\left[\boldsymbol{t}_{3}-\boldsymbol{t}_{\mathbf{4}}\right]$ : At time $t_{3}, v_{\text {Coss } 2}=v_{\text {Coss } 4}=0$. Since $i_{L r 1}\left(t_{3}\right)>0$, $i_{L r 2}\left(t_{3}\right)>0$ and $i_{L r 3}\left(t_{3}\right)>0$, the anti-parallel diodes of $S_{2}$ and $S_{4}$ are conducting. $S_{2}$ and $S_{4}$ can be turned on at this moment under ZVS. In mode $4, D_{2}$ and $D_{4}$ are conducting, $i_{L m 1}-i_{L m 4}$ decrease. In addition, $v_{C o s s 1}=v_{C r 1}+v_{C r 2}, v_{C o s s 3}=v_{C r 3}+v_{C r 4}$, and $v_{C r 5}+v_{C r 6}=v_{C r 3}+v_{C r 4} . L_{r 1}, C_{r 1}$ and $C_{r 2}$ are resonant in circuit 1; $L_{r 2}, C_{r 3}$ and $C_{r 4}$ are resonant in circuit 2; $L_{r 3}, C_{r 5}$ and $C_{r 6}$ are resonant in circuit 3; and power is transferred from the input voltage $V_{\text {in }}$ to the output load $R_{o}$. The resonant frequency is $f_{r}=1 / 2 \pi \sqrt{2 L_{r} C_{r}}$.

Mode $5\left[\boldsymbol{t}_{\mathbf{4}}-\boldsymbol{t}_{5}\right]$ : At time $t_{4}, i_{L r 1}\left(t_{4}\right)=i_{L m 1}\left(t_{4}\right), i_{L r 2}\left(t_{4}\right)=i_{L m 2}\left(t_{4}\right)$ and $i_{L r 3}\left(t_{4}\right)=i_{L m 3}\left(t_{4}\right)=i_{L m 4}\left(t_{4}\right)$. Diodes $D_{1}-D_{4}$ are all in the off-state. $L_{r 1}, L_{m 1}, C_{r 1}$ and $C_{r 2}$ are resonant in circuit $1 ; L_{r 2}$, $L_{m 2}, C_{r 3}$ and $C_{r 4}$ are resonant in circuit $2 ; L_{r 3}, L_{m 3}, L_{m 4}, C_{r 5}$ and $C_{r 6}$ are resonant in circuit 3; and the resonant frequency is $f_{p}=1 / 2 \pi \sqrt{2\left(L_{r}+L_{m}\right) C_{r}}$.

Mode $6\left[\boldsymbol{t}_{5}-\boldsymbol{T}+\boldsymbol{t}_{0}\right]$ : At time $t_{5}, S_{2}$ and $S_{4}$ are turned off. Diodes $D_{1}$ and $D_{3}$ are conducting. The magnetizing voltages $v_{L m 1}-v_{L m 4}$ are positive and the magnetizing currents $i_{L m 1}-$ $i_{L m 4}$ increase. Since $i_{L r 1}\left(t_{5}\right)<0, i_{L r 2}\left(t_{5}\right)<0$ and $i_{L r 3}\left(t_{5}\right)<0, C_{o s s 1}$ and $C_{\text {oss } 3}$ are discharged and $C_{\text {oss } 2}$ and $C_{\text {oss } 4}$ are charged. $C_{\text {oss } 1}$ and $C_{\text {oss3 }}$ can be discharged to zero voltage if the energy stored in $L_{r 1}-L_{r 3}$ at $t_{5}$ is greater than the energy stored in $C_{\text {oss } 1}-C_{\text {oss } 4}$. Then, the operating modes of the proposed converter in a switching period are completed.

\section{CONVERTER PERFORMANCE ANALYSIS AND DESIGN EXAMPLE}

Three resonant circuits are included in the proposed converter to share the load power. The power transferred through the three resonant circuits is a function of the switching frequency. The input terminal of the resonant circuit is a square wave voltage. If the bandwidth of the resonant circuit is less than the switching frequency, the harmonics of the input square wave voltage can be neglected at the output terminal. The secondary side currents $i_{D 1}+i_{D 2}$ and $i_{D 3}+i_{D 4}$ are quasi-sinusoidal currents. If the primary inductor currents are greater than the magnetizing currents, then diodes $D_{1}$ and $D_{3}$ are conducting and $v_{L m 1}=v_{L m 2}=n V_{o} / 2$ and $v_{L m 3}=v_{L m 4}=n V_{o} / 4$. On the other hand, $v_{L m 1}=v_{L m 2}=-n V_{o} / 2$, $v_{L m 3}=v_{L m 4}=-n V_{o} / 4$ and rectifier diodes $D_{2}$ and $D_{4}$ are conducting when the primary inductor currents are less than the magnetizing currents. Since the charge/discharge time of output capacitors $C_{\text {oss } 1}-C_{\text {oss } 4}$ in modes 3 and 6 and the time intervals in modes 2 and 5 are much less than the time intervals in modes 1 and 4 , the magnetizing inductor voltages $v_{L m 1}-v_{L m 4}$ approximate quasi-square waveforms.

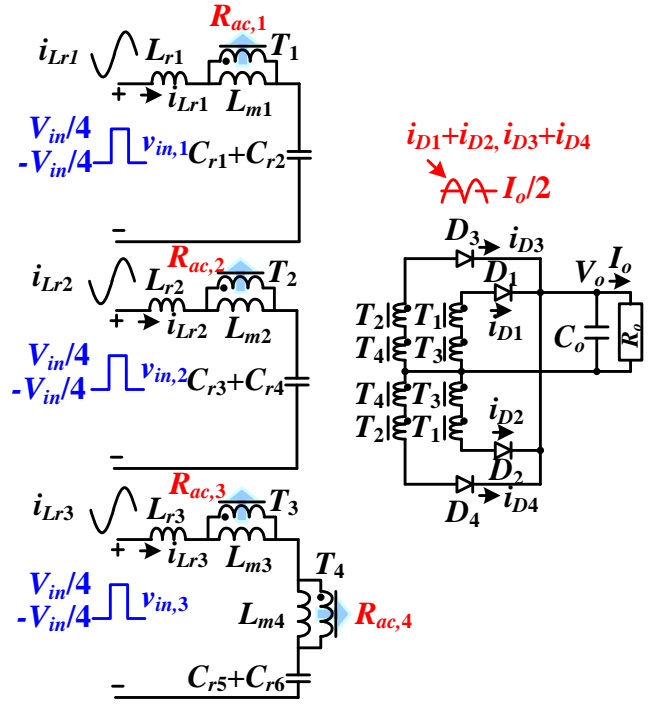

Fig. 4. Equivalent circuit of the proposed converter for the derivation of steady state model.

$$
\begin{aligned}
& v_{L m 1}=v_{L m 2}=\sum_{m=1,3,5 \ldots} \frac{2 n V_{o}}{m \pi} \sin \left(2 \pi m f_{s} t-\theta_{m}\right) \\
& v_{L m 3}=v_{L m 4}=\sum_{m=1,3,5 \ldots} \frac{n V_{o}}{m \pi} \sin \left(2 \pi m f_{s} t-\theta_{m}\right)
\end{aligned}
$$

where $\theta_{m}$ is the phase angle of the $m$-th harmonic frequency. The peak secondary winding currents are given as:

$$
\hat{i}_{T 1, \mathrm{sec}}=\hat{i}_{T 2 \text { sec }}=\hat{i}_{T 3, \mathrm{sec}}=\hat{i}_{T 4, \mathrm{sec}}=\pi I_{o} / 4
$$

Thus, the load resistance $R_{o}$ reflected to the transformer primary sides is shown as:

$$
\begin{aligned}
& R_{a c, 1}=R_{a c, 2}=\frac{\hat{v}_{L m 1, f}}{\hat{i}_{T 1, \mathrm{sec}} / n}=\frac{8 n^{2}}{\pi^{2}} R_{o} \\
& R_{a c, 3}=R_{a c, 4}=\frac{\hat{v}_{L m 3, f}}{\hat{i}_{T 3, \mathrm{sec}} / n}=\frac{4 n^{2}}{\pi^{2}} R_{o}
\end{aligned}
$$

Fig. 4 shows the AC resonant circuits excited by the effective sinusoidal input voltage and the effective resistive loads $R_{a c, 1}-R_{a c, 4}$. The input impedances $Z_{i n, 1}-Z_{i n, 3}$ of the resonant circuits are expressed as:

$$
\begin{aligned}
& Z_{i n, 1}\left(f_{s}\right)=Z_{i n, 2}\left(f_{s}\right) \\
& =\frac{R_{a c, 1}\left(j 2 \pi f_{s} L_{m, 1}\right)}{R_{a c, 1}+j 2 \pi f_{s} L_{m, 1}}+j 2 \pi f_{s} L_{r 1}+\frac{1}{j 2 \pi f_{s}\left(C_{r 1}+C_{r 2}\right)}(6) \\
& =\frac{R_{a c, 1}\left(j 2 \pi f_{s} L_{m}\right)}{R_{a c, 1}+j 2 \pi f_{s} L_{m}}+j 2 \pi f_{s} L_{r}+\frac{1}{j 2 \pi f_{s}\left(2 C_{r}\right)} \\
& Z_{i n, 3}\left(f_{s}\right)==Z_{i n, 1}\left(f_{s}\right)=Z_{i n, 2}\left(f_{s}\right) \\
& =\frac{\left(R_{a c, 3}+R_{a c, 4}\right) j 2 \pi f_{s}\left(L_{m 3}+L_{m 4}\right)}{\left(R_{a c, 3}+R_{a c, 4}\right)+j 2 \pi f_{s}\left(L_{m 3}+L_{m 4}\right)}+j 2 \pi f_{s} L_{r 3}+\frac{1}{j 2 \pi f_{s}\left(C_{r 5}+C_{r 6}\right)} \\
& =\frac{R_{a c, 1}\left(j 2 \pi f_{s} L_{m}\right]}{R_{a c, 1}+j 2 \pi f_{s} L_{m}}+j 2 \pi f_{s} L_{r}+\frac{1}{j 2 \pi f_{s}\left(2 C_{r}\right)}
\end{aligned}
$$


The frequency modulation (FM) approach is adopted to regulate the AC voltage gain of the resonant circuit. The AC voltage gain of the resonant circuit is approximately expressed as:

$$
\left|G_{a c}(f)\right|=1 / \sqrt{\left[1+k\left(1-\frac{f_{r}^{2}}{f_{s}^{2}}\right)\right]^{2}+Q^{2}\left(\frac{f_{s}}{f_{r}}-\frac{f_{r}}{f_{s}}\right)^{2}}
$$

where $Q=\sqrt{L_{r} /\left(2 C_{r}\right)} / R_{a c_{,} 1}, \quad f_{r}=1 /\left(2 \pi \sqrt{2 L_{r} C_{r}}\right)$, $k=L_{r} / L_{m}$ and $f_{s}$ is the switching frequency. The DC voltage gain $G_{d c}$ of the proposed converter is given as $G_{d c}=2 n V_{o} / V_{i n}$. The AC voltage gain at the no-load condition $(Q=0)$ and $f_{s}=\infty$ is given as $\left|G_{a c}(f)\right|_{N f_{s}=\infty}=1 /(1+k)$. In order to regulate the output voltage from no-load to full load, the minimum DC voltage gain must be greater than the AC voltage gain at the no-load condition. Thus, the minimum turns ratio of transformers $T_{1}$ and $T_{2}$ is given in (9).

$$
n_{\min } \geq \frac{V_{i n, \max }}{2 V_{o}(1+k)}
$$

A design example of the prototype circuit is provided in order to verify the system analysis of the proposed converter. A laboratory prototype was constructed to verify the effectiveness of the proposed converter. The electric specifications are $V_{\text {in }}=750-800 \mathrm{~V}, V_{o}=48 \mathrm{~V}$, and $I_{o \text {,rated }}=40 \mathrm{~A}$. The selected series resonant frequency $f_{r}$ is $120 \mathrm{kHz}$. The selected inductance ratio $L_{m} / L_{r}=1 / k=7$.

\section{A. Turns Ratio of $T_{1}-T_{4}$}

In the prototype circuit, the DC voltage gain is equal to $G_{d c}=2 n V_{o} / V_{i n}$. Thus, the minimum turns ratio is given as $\boldsymbol{n}=\boldsymbol{G}_{\boldsymbol{d}, \boldsymbol{\operatorname { m i n }}} \boldsymbol{V}_{\boldsymbol{i n , \operatorname { m i n } \boldsymbol { x }}} /\left(2 \boldsymbol{V}_{\boldsymbol{o}}\right)$. If the minimum DC voltage gain is selected as unity at the series resonant frequency, then the turns ratio of $T_{1}-T_{4}$ is given as:

$$
\begin{aligned}
& n_{1}=n_{2}=\frac{n_{p}}{n_{s}}=\frac{G_{d c, \min } V_{i n, \max }}{2 V_{o}}=\frac{1 \times 800}{2 \times 48}=8.33 \\
& n_{3}=n_{4}=\frac{n_{1}}{2}=4.166
\end{aligned}
$$

The actual primary and secondary turns used in $T_{1}-T_{4}$ are $n_{p, T 1}=n_{p, T 2}=66$ turns, $n_{s, T 1}=n_{s, T 1}=8$ turns, $n_{p, T 3}=n_{p, T 4}=33$ turns and $n_{s, T 3}=n_{s, T 4}=8$ turns. The actual turns ratios of $T_{1}$ and $T_{2}$ are 8.25, and the turns ratios of $T_{3}$ and $T_{4}$ are 4.125.

\section{B. DC Voltage Gain of the Proposed Converter}

Based on the selected turns ratio of $T_{1}-T_{4}$, the minimum and maximum DC voltage gains of the proposed converter are derived as:

$$
\begin{aligned}
& G_{d c, \min }=\frac{2 n V_{o}}{V_{i n, \max }}=\frac{2 \times(66 / 8) \times 48}{800}=0.99 \\
& G_{d c, \max }=\frac{2 n V_{o}}{V_{i n, \min }}=\frac{2 \times(66 / 8) \times 48}{750}=1.056
\end{aligned}
$$

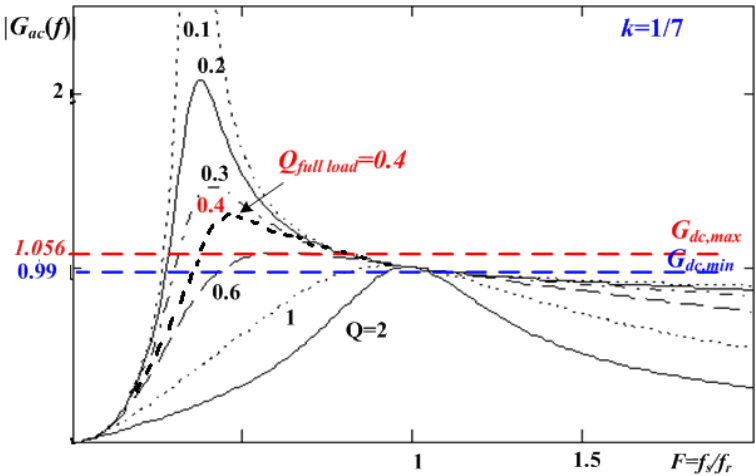

Fig. 5. AC voltage gain and DC voltage gain at different frequency ratio $f_{s} / f_{r}$.

\section{Q Value at Full Load and AC Equivalent Resistance}

Fig. 5 gives the AC voltage gain versus the frequency ratio $f_{s} / f_{r}$ with $k=1 / 7$. Since the minimum and maximum DC gains are 0.99 and 1.056, respectively, the maximum $Q$ at a full load should be less than 0.4 in order to effectively regulate the output voltage. In this prototype, the $Q$ value at a full load is selected as 0.4. Based on (4) and (5), the AC equivalent resistances $R_{a c, 1}-R_{a c, 4}$ at a full load are derived as:

$$
\begin{aligned}
& R_{a c, 1}=R_{a c, 2}=\frac{8 n^{2}}{\pi^{2}} R_{o}=\frac{8 \times(66 / 8)^{2}}{3.14159^{2}} \times \frac{48}{40} \approx 66.2 \Omega \\
& R_{a c, 3}=R_{a c, 4}=\frac{4 n^{2}}{\pi^{2}} R_{o}=\frac{4 \times(66 / 8)^{2}}{3.14159^{2}} \times \frac{48}{40} \approx 33.1 \Omega
\end{aligned}
$$

\section{Resonant Capacitances and Inductances}

Since $f_{r}=1 / 2 \pi \sqrt{2 L_{r} C_{r}}$ and $Q=\sqrt{L_{r} /\left(2 C_{r}\right)} / R_{\alpha c_{,}, 1}$, the resonant capacitances $C_{r 1}-C_{r 6}$ and inductances $L_{r 1}-L_{r 3}$ are obtained as:

$$
\begin{aligned}
& L_{r 1}=L_{r 2}=L_{r 3}=L_{r}=\frac{Q R_{\alpha c, 1}}{2 \pi f_{r}}=\frac{0.4 \times 66.2}{2 \pi \times 120000} \approx 35 \mu \mathrm{H} \\
& C_{r 1}=\ldots=C_{r 6}=C_{r} \\
& =\frac{1}{8 \pi^{2} L_{r} f_{r}^{2}}=\frac{1}{8 \pi^{2} \times 35 \times 10^{-6} \times(120000)^{2}} \approx 25 \mathrm{nF}
\end{aligned}
$$

The magnetizing inductances of $T_{1}-T_{4}$ are expressed as:

$$
\begin{aligned}
& L_{m 1}=L_{m 2}=L_{r} / k=\frac{35 \mu H}{1 / 7}=245 \mu \mathrm{H} \\
& L_{m 3}=L_{m 4}=L_{m 1} / 2=122.5 \mu \mathrm{H}
\end{aligned}
$$

\section{E. Power Semiconductors}

The input maximum voltage is $800 \mathrm{~V}$ and the voltage stresses of $S_{1}-S_{4}$ are equal to $V_{\text {in }} / 2=400 \mathrm{~V}$. Power MOSFETs (IRFP460) with a $500 \mathrm{~V}$ voltage rating and a 13 A current rating at $100^{\circ} \mathrm{C}$ are used for power switches $S_{1}-S_{4}$. The output voltage is $48 \mathrm{~V}$ and the load current is $40 \mathrm{~A}$. The average currents of $D_{1}-D_{4}$ are equal to $40 \mathrm{~A} / 4=10 \mathrm{~A}$. The voltage stresses of $D_{1}-D_{4}$ are equal to $2 V_{o}=96 \mathrm{~V}$. Fast 


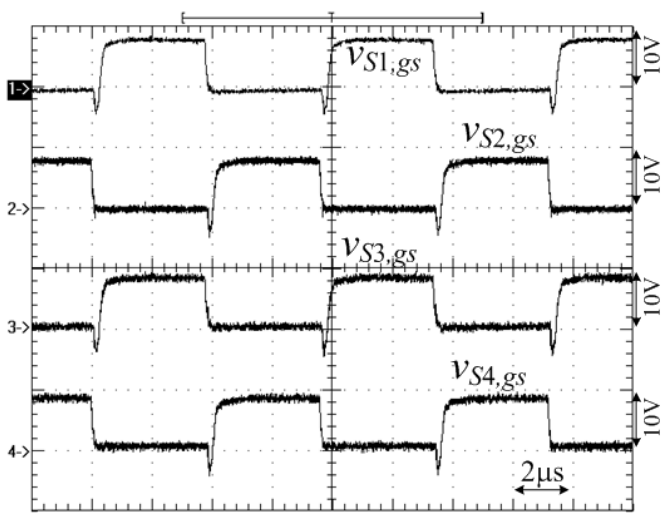

(a)

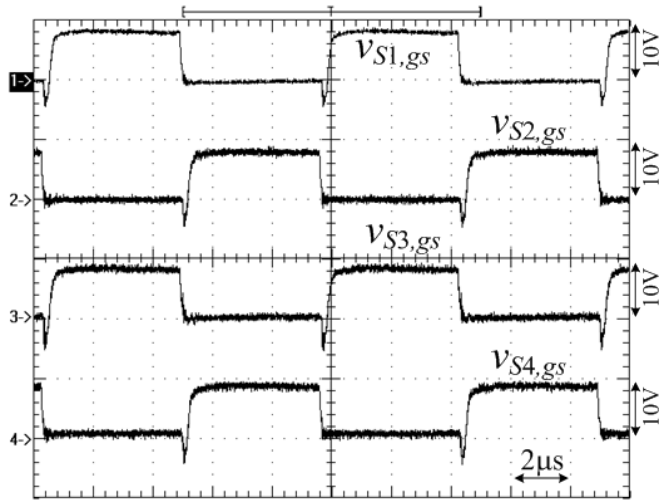

(b)

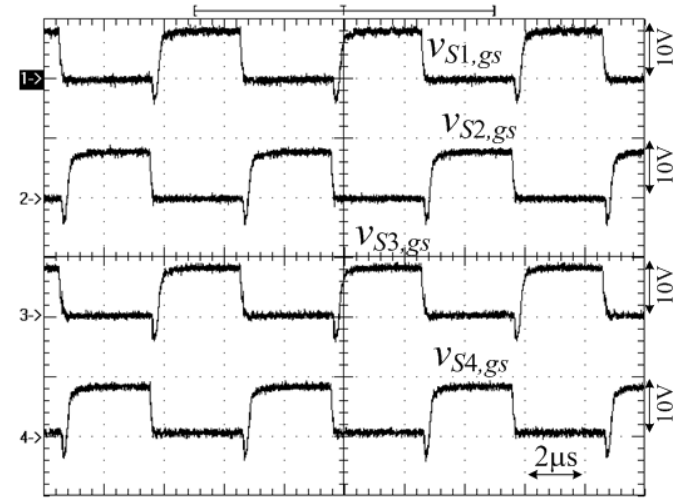

(c)

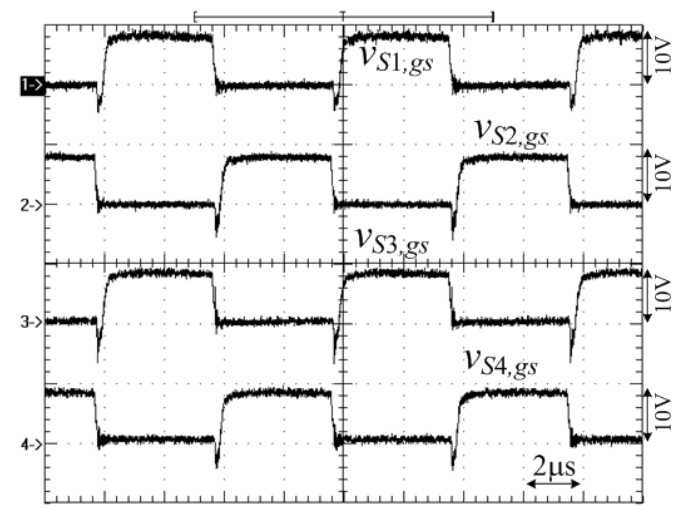

(d)

Fig. 6. Measured PWM waveforms of $S_{1}-S_{4}$ at (a) $V_{\text {in }}=750 \mathrm{~V}$ and $25 \%$ load. (b) $V_{\text {in }}=750 \mathrm{~V}$ and $100 \%$ load. (c) $V_{\text {in }}=800 \mathrm{~V}$ and $25 \%$ load. (d) $V_{i n}=800 \mathrm{~V}$ and $100 \%$ load.

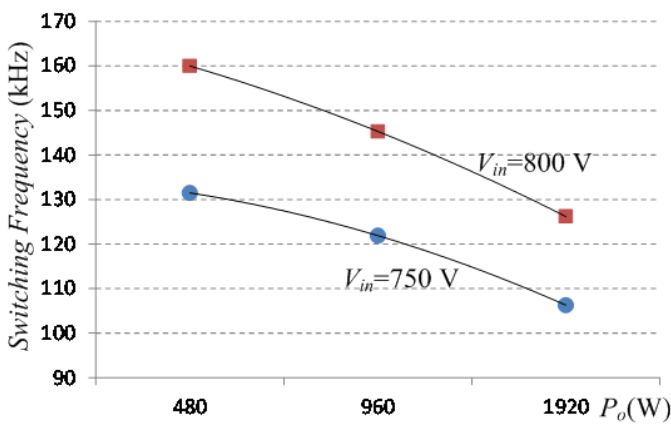

Fig. 7. Measured switching frequencies at different input voltages and load conditions.

recovery diodes (KCU30A20) with a $200 \mathrm{~V}$ voltage rating and a $30 \mathrm{~A}$ current rating are adopted for $D_{1}-D_{4}$ in the prototype circuit.

\section{EXPERIMENTAL RESULTS}

Based on the derived circuit parameters in the previous section, experimental verification is provided to demonstrate the performance of the proposed circuit. The measured PWM waveforms of $S_{1}-S_{4}$ at different input voltages and load conditions are given in Fig. 6. $S_{1}$ and $S_{3}$ have the same PWM waveforms, and $S_{2}$ and $S_{4}$ have identical PWM signals. However, the PWM signals of $S_{1}$ and $S_{2}$ are complementary each other to avoid short circuits at each half-bridge leg. At the same load power, the switching frequency at a low input voltage $V_{\text {in }}=750 \mathrm{~V}$ is less than the switching frequency at a high input voltage $V_{i n}=800 \mathrm{~V}$. At the same input voltage, $V_{\text {in }}=800 \mathrm{~V}$, the switching frequency at a full load is less than the switching frequency at a light load. Fig. 7 gives the measured switching frequency of the proposed converter at different input voltages and load conditions. The measured waveforms of the gate voltage and drain voltage of $S_{1}$ and $S_{2}$ at different input voltages and load conditions are illustrated in Fig. 8. In the same manner, Fig. 9 gives the test results of the gate voltage and drain voltage of $S_{3}$ and $S_{4}$ at different input voltages and load conditions. Before $S_{1}-S_{4}$ are turned on, the drain voltages are decreased to zero voltage. Therefore, the ZVS turn-on of $S_{1}-S_{4}$ is achieved. Fig. 10 shows the test waveforms of $i_{L r 1}-i_{L r 3}$ at a full load and different input voltages. It is clear that inductor currents $i_{L r 1}$ and $i_{L r 2}$ are balanced. Fig. 11(a) shows the test waveforms of $v_{C r 1}-v_{C r 6}$ at a full load and $V_{\text {in }}=750 \mathrm{~V}$. It is clear that $v_{C r 1}$, $v_{C r 3}$ and $v_{C r 6}$ have the same voltage waveforms and that the voltage waveforms of $v_{C r 2}, v_{C r 4}$ and $v_{C r 5}$ are balanced. Fig. 11(b) shows the test results of $v_{C r 1}+v_{C r 2}, v_{C r 3}+v_{C 4}$ and $v_{C r 5}+v_{C r 6}$. From the test results in Fig. 11(b), it can be seen that the three voltages $v_{C r 1}+v_{C r 2}, v_{C r 3}+v_{C r 4}$ and $v_{C r 5}+v_{C r 6}$ are balanced. In the same manner, the measured voltages $v_{C r 1}-v_{C r 6}, v_{C r 1}+v_{C r 2}, v_{C r 3}+v_{C r 4}$ and $v_{C r 5}+v_{C r 6}$ at $V_{i n}=800 \mathrm{~V}$ are shown in Fig. 12. Fig. 13 shows the measured gate voltage $v_{S 1, g s}$ and the output diode currents $i_{D 1}-i_{D 4}$ at a full load and 


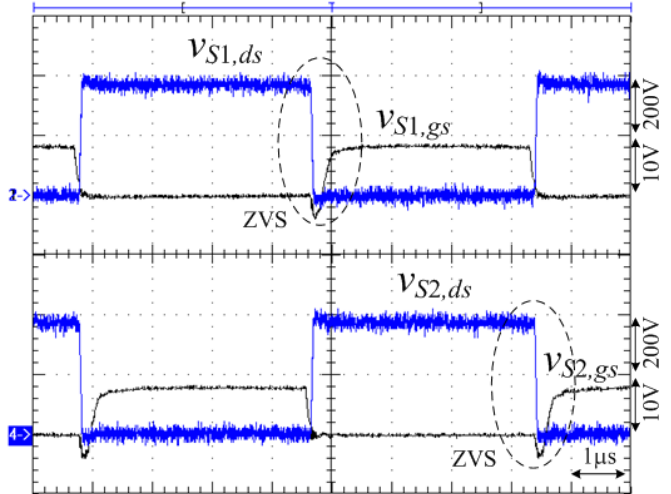

(a)

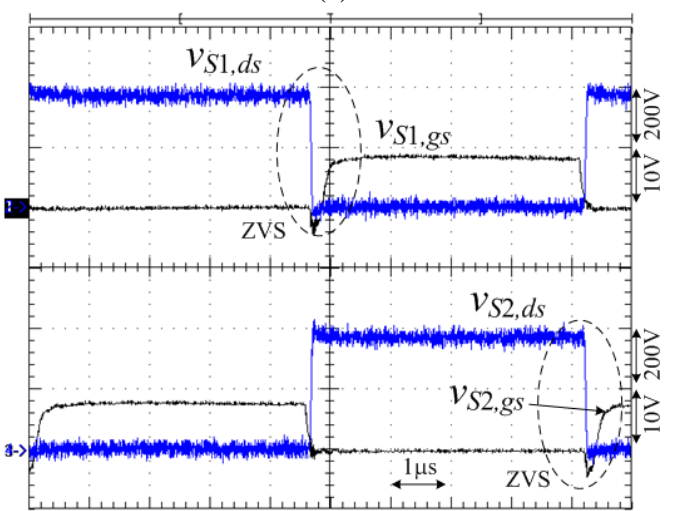

(b)

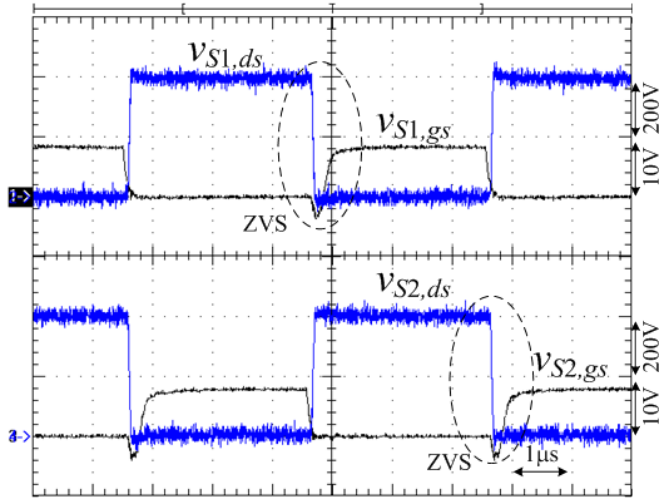

(c)

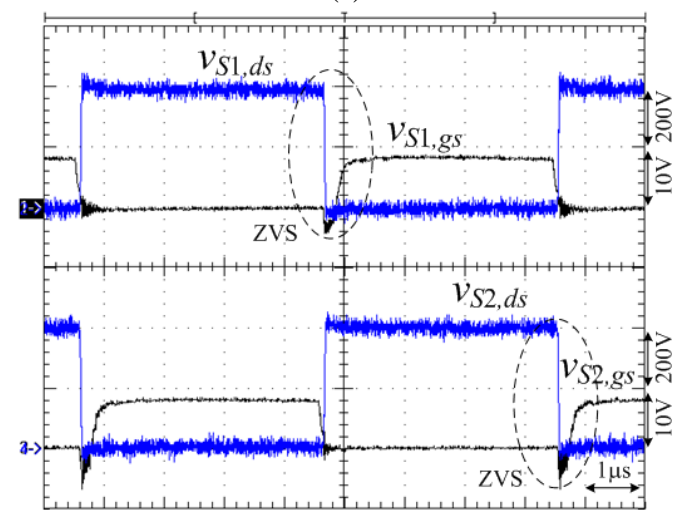

(d)

Fig. 8. Measured results of gate voltage and drain voltage of active switches. (a) $S_{1}$ and $S_{2}$ at $25 \%$ load with $V_{\text {in }}=750 \mathrm{~V}$. (b) $S_{1}$ and $S_{2}$ at $100 \%$ load with $V_{\text {in }}=750 \mathrm{~V}$. (c) $S_{1}$ and $S_{2}$ at $25 \%$ load with $V_{\text {in }}=800 \mathrm{~V}$. (d) $S_{1}$ and $S_{2}$ at $100 \%$ load with $V_{\text {in }}=800 \mathrm{~V}$.

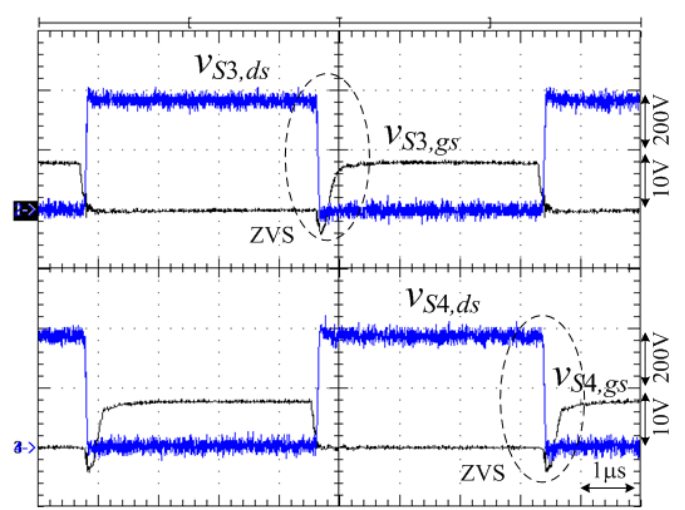

(a)

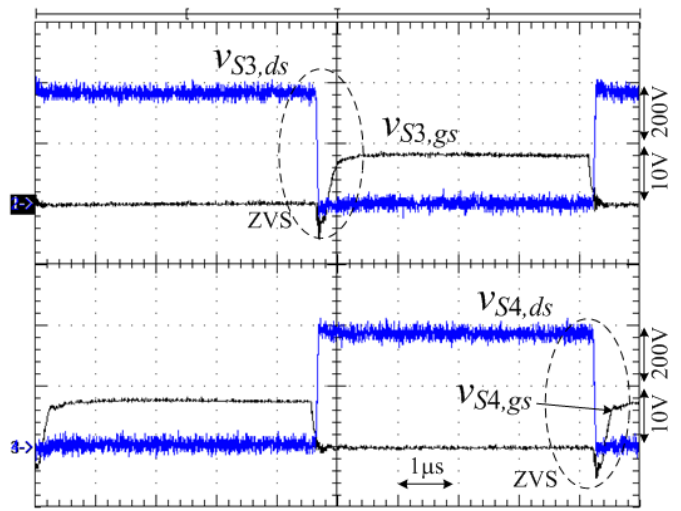

(b)

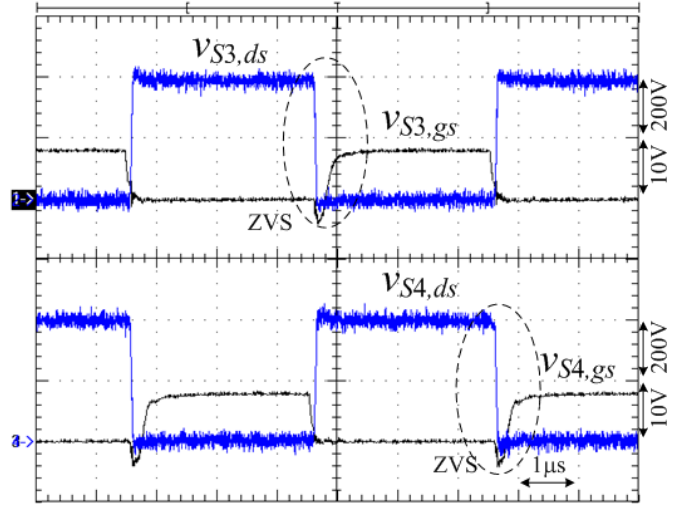

(c)

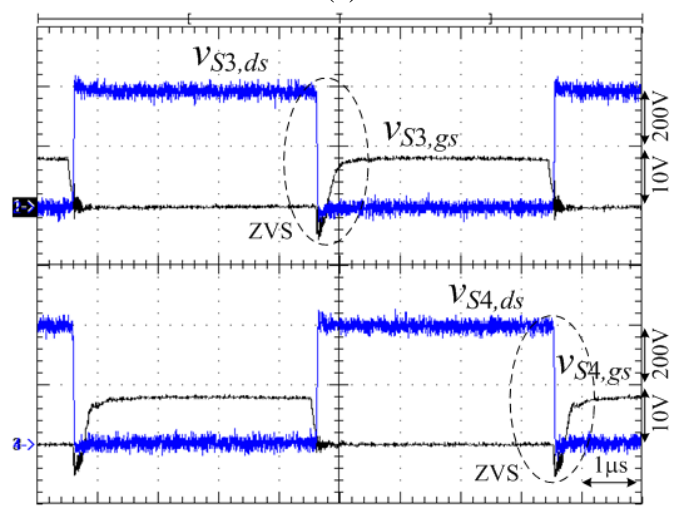

(d)

Fig. 9. Measured results of gate voltage and drain voltage of active switches. (a) $S_{3}$ and $S_{4}$ at $25 \%$ load with $V_{\text {in }}=750 \mathrm{~V}$. (b) $S_{3}$ and $S_{4}$ at $100 \%$ load with $V_{\text {in }}=750 \mathrm{~V}$. (c) $S_{3}$ and $S_{4}$ at $25 \%$ load with $V_{\text {in }}=800 \mathrm{~V}$. (d) $S_{3}$ and $S_{4}$ at $100 \%$ load with $V_{\text {in }}=800 \mathrm{~V}$. 


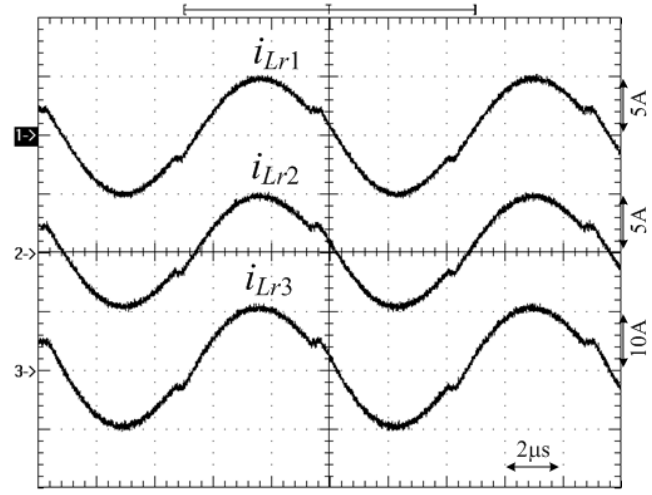

(a)

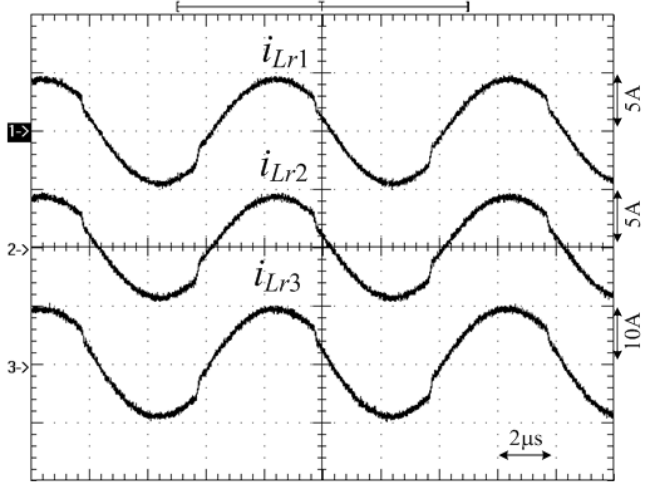

(b)

Fig. 10. Measured results of the resonant inductor currents $i_{L r 1}-i_{L r 3}$ at full load and (a) $V_{i n}=750 \mathrm{~V}$. (b) $V_{\text {in }}=800 \mathrm{~V}$.

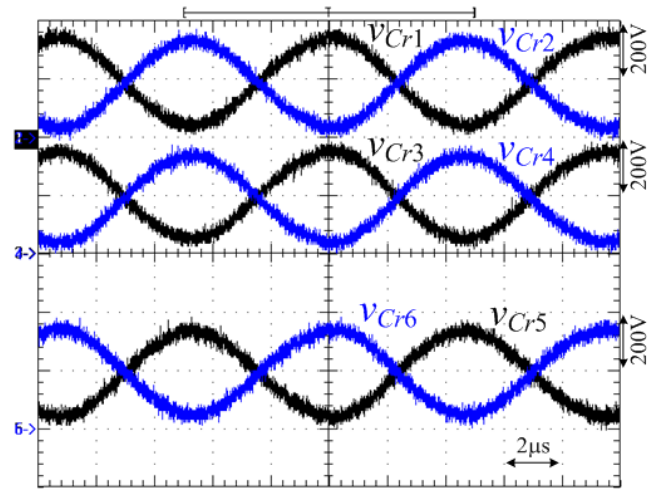

(a)

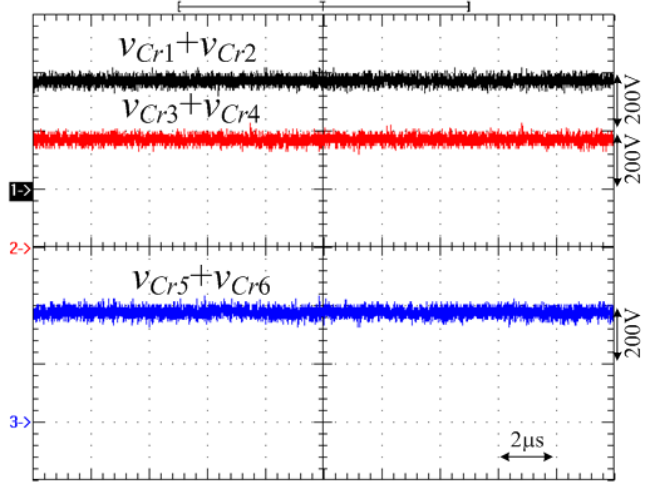

(b)

Fig. 11. Measured capacitor voltage waveforms of $C_{r 1}-C_{r 6}$ at $V_{\text {in }}=750 \mathrm{~V}$ and full load. (a) $v_{C r 1}-v_{C r 6}$. (b) $v_{C r 1}+v_{C r 2}, v_{C r 3}+v_{C r 4}$ and $v_{C r 5}+v_{C r 6}$.

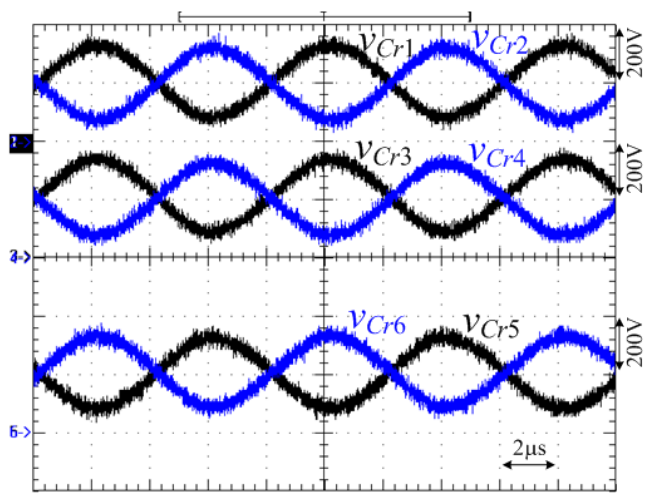

(a)

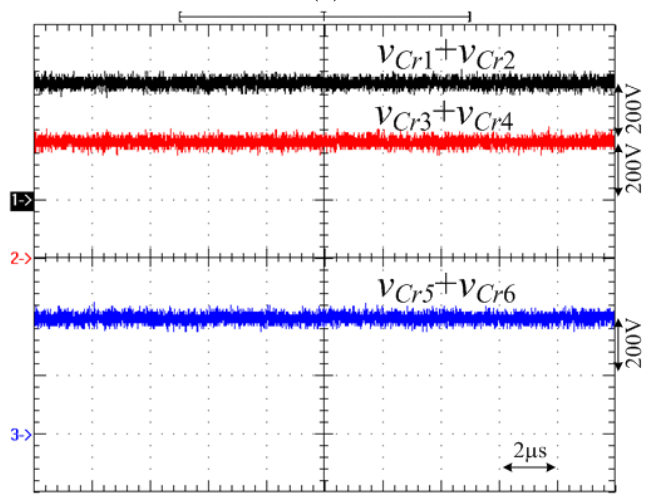

(b)

Fig. 12. Measured capacitor voltage waveforms of $C_{r 1}-C_{r 6}$ at $V_{i n}=800 \mathrm{~V}$ and full load. (a) $v_{C r 1}-v_{C r 6}$. (b) $v_{C r 1}+v_{C r 2}, v_{C r 3}+v_{C r 4}$ and $v_{C r 5}+v_{C r 6}$.

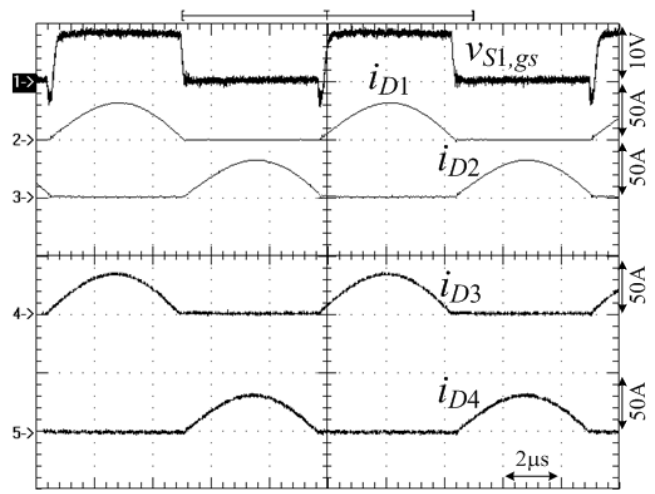

(a)

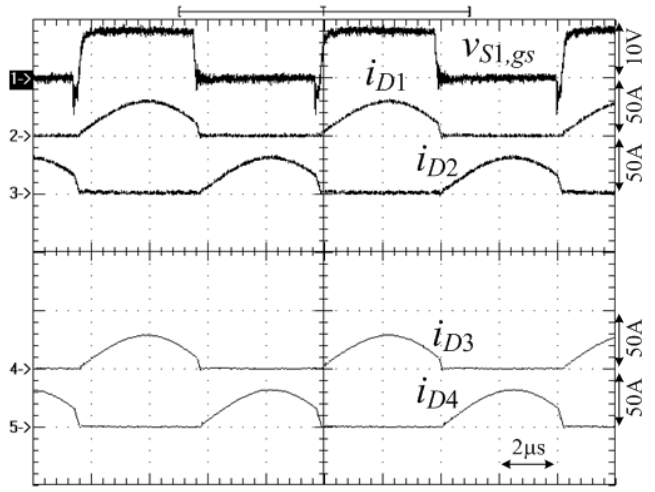

(b)

Fig. 13. Measured output diode currents at full load under (a) $V_{\text {in }}=750 \mathrm{~V}$ (b) $V_{\text {in }}=800 \mathrm{~V}$. 


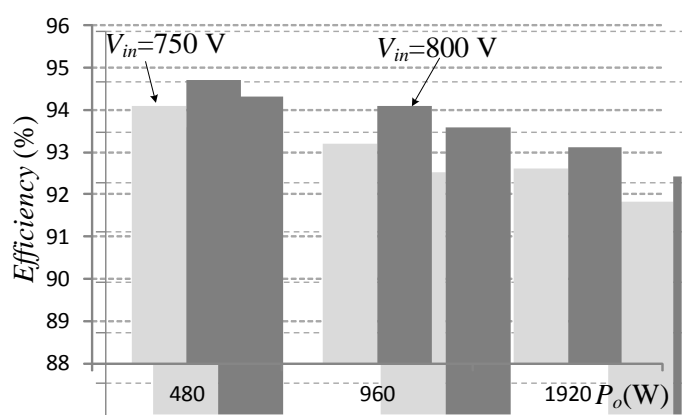

Fig. 14. Measured efficiencies at different input voltages and load conditions.

different input voltages. The diode currents $i_{D 1}$ and $i_{D 3}$ are balanced, and $i_{D 2}$ and $i_{D 4}$ are also balanced. The measured circuit efficiencies at different loads are shown in Fig. 14. The measured efficiency is greater than $92 \%$ from a $25 \%$ load to a full load.

\section{CONCLUSION}

A new soft switching DC/DC converter with balanced diode currents at the output side and low voltage stress of the power MOSFETs is presented for medium voltage applications. Three resonant circuits are adopted at the primary side and a series-connection of isolation transformers at the secondary side in order to balance the output diode currents of the proposed converter. The power rating of each resonant circuit is equal to half of the load power so that the current stresses of the passive components and transformer windings are reduced. Two half-bridge circuits with four spilt capacitors are adopted so that the voltage stress of the power MOSFETs are clamped at half of the input voltage. Two resonant capacitors $C_{r 5}$ and $C_{r 6}$ are also adopted to balance the input split capacitor voltages. When compared to conventional parallel three-level converters, the proposed converter has a lower power switch count. Finally, experiments with a $1.92 \mathrm{~kW}$ prototype are provided to demonstrate the performance of the converter.

\section{ACKNOWLEDGMENT}

This project is supported by the National Science Council of Taiwan under Grant NSC 102-2221-E-224 -022 -MY3.

\section{REFERENCES}

[1] S.-Y. Cho, I.-O. Lee, J.-K. Kim, and G.-W. Moon, “A new standby structure based on a forward converter integrated with a phase-shift full-bridge converter for server power supplies," IEEE Trans. Power Electron., Vol. 28, No. 1, pp. 336-346, Jan. 2013.

[2] Z. Guo, D. Sha, X. Liao, and J. Luo, "Input-series-output-parallel phase-shift full-bridge derived DC-DC converters with auxiliary LC networks to achieve wide zero-voltage switching range," IEEE Trans. Power
Electron., Vol. 29 , No. 10, pp. 5081-5086, Oct. 2014.

[3] B. Gu, J.-S. Lai, N. Kees, and C. Zheng, "Hybrid-switching full-fridge DC-DC converter with minimal voltage stress of bridge rectifier, reduced circulating losses, and filter requirement for electric vehicle battery chargers," IEEE Trans. Power Electron., Vol. 28, No. 3, pp. 1132-1144, Mar. 2013.

[4] Y. Jiang, Z. Chen, and J. Pan, "Zero-voltage switching phase shift full-bridge step-up converter with integrated magnetic structure," IET Proceedings -Power Electron., Vol. 3, No. 5, pp. 732-739, 2010.

[5] B. M. Song, R. McDowell, A. Bushnell, and J. Ennis, “A three-level DC-DC converter with wide input voltage operations for ship-electric-power-distribution systems," IEEE Trans. Plasma Science. Vol. 32, No. 5, pp. 1856-1863, Oct. 2004.

[6] A. D. Cheok, S. Kawamoto, T. Matsumoto, and H. Obi, "High power AC/DC and DC/AC inverter for high speed train," in Proc. of IEEE TENCON Conf., pp. 423-428, 2000.

[7] U. Drofenik and F. Canales, "European trends and technologies in traction," in Proc. of IPEC Conf., 2014.

[8] J. R. Pinheiro and I. Barbi, "The three-level ZVS-PWM DC-to-DC converter," IEEE Trans. Power Electron., Vol. 8, No. 4, pp. 486-492, Oct. 1993.

[9] F. Canales, P. Barbosa, and F. C. Lee, "A zero-voltage and zero-current switching three-level DC/DC converter," IEEE Trans. Power Electron., Vol. 17, No. 6, pp. 898-904, Nov. 2002.

[10] Y. Gu, Z. Lu, L. Hang, Z. Qian, and G. Huang, “Three-level LLC series resonant DC/DC converter," IEEE Trans. Power Electron., Vol. 20, No. 4, pp. 781-789, Jul. 2005.

[11] Y. Shi and X. Yang, "Zero-voltage switching PWM three-level full-bridge DC-DC converter with wide ZVS load range,” IEEE Trans. Power Electron., Vol. 28 , No. 10, pp. 4511-4524, Oct. 2013.

[12] J. L. Duarte, J. Lokos, and F. B. M. van Horck, "Phase-shift-controlled three-level converter with reduced voltage stress featuring ZVS over the full operation range," IEEE Trans. Power Electron., Vol. 28, No. 5, pp. 2140-2150, May 2013.

[13] W. Chen and X. Ruan, "Zero-voltage-switching PWM hybrid full-bridge three-level converter with secondary-voltage clamping scheme,” IEEE Trans. Ind. Electron., Vol. 55, No. 2, pp. 644-654, Feb. 2008.

[14] J. P. Rodrigues, S. A. Mussa, I. Barbi, and A. J. Perin, "Three-level zero-voltage switching pulse-width modulation DC-DC boost converter with active clamping," IET Proc.- Power Electron., Vol. 3, No. 3, pp. 345-354, 2010.

[15] B. Yang, F. C. Lee, A. J. Zhang, and G. Huang, "LLC resonant converter for front end DC/DC conversion," in Proc. of IEEE APEC, Vol. 2, pp. 1108-1112, 2002.

[16] R. Beiranvand, B. Rashidian, M. R. Zolghadri and S. M. H. Alavi, "Using LLC resonant converter for designing wide-range voltage source," IEEE Trans. Ind. Electron., Vol. 58, No. 5, pp. 1746-1756, May 2011.

[17] D. Fu, Y. Liu, F. C. Lee, and M. Xu, "A novel driving scheme for synchronous rectifiers in LLC resonant converters,” IEEE Trans. Power Electron., Vol. 24, No. 5, pp. 1321-1329, May 2009.

[18] R. Beiranvand, B. Rashidian, M. R. Zolghadri and S. M. H. Alavi, “A design procedure for optimizing the LLC resonant 
converter as a wide output range voltage source," IEEE Trans. Power Electron., Vol. 27, No. 8, pp. 3749-3763, Apr. 2012.

[19] B.-R. Lin and C.-C. Chien, "Analysis and implementation of a new soft switching DC/DC PWM converter," IET Proc.-Power Electron., Vol. 6, No. 1, pp. 202-213, 2013.

[20] B.-R. Lin and H.-Y. Shih, "ZVS converter with parallel connection in primary side and series connection in secondary side,” IEEE Trans. Ind. Electron., Vol. 58, No. 4, pp. 1251-1258, Apr. 2011.

[21] B.-R. Lin and P.-J. Cheng, "New ZVS DC--DC converter with series-connected transformers to balance the output currents,” IEEE Trans. Power Electron., Vol. 29, No. 1, pp. 246-255, Jan. 2014.

[22] C. Dong and Z. P. Fang, "A family of zero current switching switched-capacitor DC-DC converters," in Proc. IEEE APEC Conf., pp. 1365-1372, 2010.

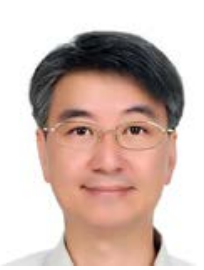

Bor-Ren Lin received his B.S. degree in Electronic Engineering from the National Taiwan University of Science and Technology, Taipei, Taiwan, in 1988, and his M.S. and Ph.D. degrees in Electrical Engineering from the University of Missouri, Columbia, MO, USA, in 1990 and 1993, respectively. From 1991 to 1993, he was a Research Assistant with the Power Electronic Research Center, University of Missouri. Since 1993, he has been with the Department of Electrical Engineering, National Yunlin University of Science and Technology, Douliou, Taiwan, where he is currently a Distinguished Professor. He is an Associate Editor of the Institution of Engineering and Technology Proceedings-Power Electronics and the Journal of Power Electronics. His current research interests include power-factor correction, multilevel converters, active power filters, and soft-switching converters. He has authored more than 200 published technical journal papers in the area of power electronics. Dr. Lin is an Associate Editor of the IEEE Transactions on Industrial Electronics. He was a recipient of Research Excellence Awards in 2004, 2005, 2007 and 2011 from the College of Engineering and the National Yunlin University of Science and Technology. He received Best Paper Awards from the 2007 and 2011 IEEE Conference on Industrial Electronics and Applications, 2007 Taiwan Power Electronics Conference, 2009 IEEE-Power Electronics and Drive Systems Conference, the 2012 Taiwan Electric Power Engineering Conference, and the 2014 IEEE-International Conference on Industrial Technology.

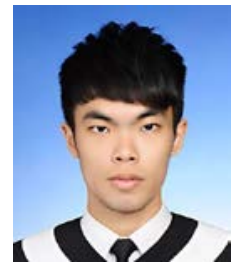

Yan-Kang Du is currently working toward his M.S. degree in Electrical Engineering at the National Yunlin University of Science and Technology, Yunlin, Taiwan (ROC). His current research interests include the design and analysis of power factor correction techniques, switching mode power supplies and soft switching converters. 